\title{
Bioorthogonal Fluorophore Linked DFO-Technology Enabling Facile Chelator Quantification and Multimodal Imaging of Antibodies
}

\author{
Labros G. Meimetis ${ }^{\dagger, \#}$, Eszter Boros ${ }^{\S,}$, ,\#, Jonathan C. Carlson ${ }^{\dagger, \#}$, Chongzhao Ran ${ }^{\S}$, Peter \\ Caravan $\S$, and Ralph Weissleder ${ }^{\dagger, \ddagger},{ }^{*}$ \\ †Center for Systems Biology, Massachusetts General Hospital, 185 Cambridge Street, CPZN \\ 5206, Boston, Massachusetts 02114, United States \\ ‡Department of Systems Biology, Harvard Medical School, 200 Longwood Avenue, Boston, \\ Massachusetts 02115, United States \\ $\S$ The Athinoula A. Martinos Center for Biomedical Imaging, Department of Radiology, \\ Massachusetts General Hospital, 149 Thirteenth street, Charlestown, Massachusetts 02129, \\ United States
}

\section{Abstract}

Herein we describe the development and application of a bioorthogonal fluorogenic chelate linker that can be used for facile creation of labeled imaging agents. The chelate linker is based on the trans-cyclooctene(TCO)-tetrazine(Tz) chemistry platform and incorporates deferoxamine (DFO) as a ${ }^{89} \mathrm{Zr}$ PET tracer and a BODIPY fluorophore for multimodal imaging. The rapid $(<3 \mathrm{~min})$ ligation between $\mathrm{mAb}-\mathrm{TCO}$ and Tz-BODIPY-DFO chelator is monitored using fluorescence and allows for determination of labeling completion. Utilizing BODIPY as the linker between $\mathrm{mAb}$ and DFO facilitates in chelator quantification using spectrophotometry, allowing for an alternative to traditional methods (mass and isotope dilution assay). Radiolabeling with ${ }^{89} \mathrm{Zr}$ to form ${ }^{89} \mathrm{Zr}$ DFO-BODIPY-trastuzumab was found to be quantitative after incubation at room temperature for $1 \mathrm{~h}(1.5 \mathrm{mCi} / \mathrm{mg}$ specific activity). The cell binding assay using HER2+ (BT474) and HER2(BT20) cell lines showed significant binding to ${ }^{89} \mathrm{Zr}$-DFO-BODIPY-trastuzumab $(6.45 \pm 1.87 \%$ in BT474 versus $1.47 \pm 0.39 \%$ in BT20). In vivo PET imaging of mice bearing BT20 or BT474 xenografts with ${ }^{89} \mathrm{Zr}$-DFO-BODIPY-trastuzumab showed high tumor conspicuity, and biodistribution confirmed excellent, specific probe uptake of $237.3 \pm 14.5 \% \mathrm{ID} / \mathrm{g}$ in BT474 xenografts compared to low, nonspecific probe uptake in BT20 xenografts $(16.4 \pm 5.6 \% \mathrm{ID} / \mathrm{g}) 96 \mathrm{~h}$ p.i. . Ex vivo fluorescence $\left(465_{\mathrm{ex}} / 520_{\mathrm{em}}\right)$ of selected tissues confirmed superb target localization

\footnotetext{
*Corresponding Authors: eboros@nmr.mgh.harvard.edu., rweissleder@mgh.harvard.edu. Phone: 617-726-8226.

\#Author Contributions

Labros G. Meimetis, Eszter Boros, and Jonathan C. Carlson contributed equally.

Notes

The authors declare no competing financial interest.

Supporting Information

The Supporting Information is available free of charge on the ACS Publications website at DOI: 10.1021/acs.bioconj-chem.5b00630. NMR and MALDI spectroscopy (PDF)
} 
and persistence of the fluorescence of ${ }^{89} \mathrm{Zr}$-DFO-BODIPY-trastuzumab. The described platform is universally adaptable for simple antibody labeling.

\section{Graphical abstract}

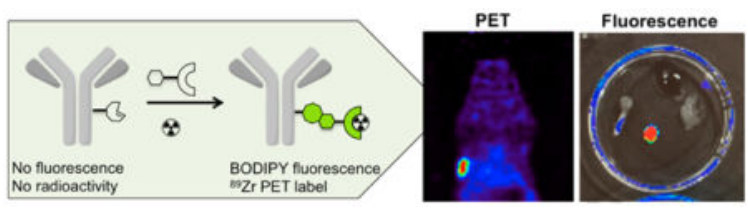

\section{INTRODUCTION}

Targeted monoclonal antibody $(\mathrm{mAb})$ therapy is a promising area of clinical medicine with an increasing number of clinically available immunotherapeutics and many in clinical and preclinical development. ${ }^{1,2}$ Due to their high target specificity, mAbs can be used in concert with positron emission tomography (PET) as a powerful noninvasive method for the direct monitoring of tumor lesions and in determining a patient's course of treatment. ${ }^{3,4}{ }^{89} \mathrm{Zr}\left(t_{1 / 2}\right.$ $\left.=78.4 \mathrm{~h}, \beta^{+}=395 \mathrm{keV}(22 \%), \gamma=897 \mathrm{keV}\right)$ is one of several ideal isotopes for this purpose and is already in use for clinical immunoPET. ${ }^{5,6}$ Attachment of ${ }^{89} \mathrm{Zr}$ to mAbs requires the use of a bifunctional metal chelator that provides stable incorporation of the radioisotope onto the mAb. Deferoxamine (DFO) is currently considered the gold standard ${ }^{89} \mathrm{Zr}$ chelator. ${ }^{7-9}$ Conjugation of DFO can be carried out using various established methods, whereafter the corresponding ${ }^{89} \mathrm{Zr}$ complex is formed rapidly under mild conditions that do not compromise the integrity of sensitive mAbs. ${ }^{10}$

In order to prepare ${ }^{89} \mathrm{Zr}$ labeled mAbs with potential for clinical use, it is imperative that there is minimal batch variation in terms of labeling efficiency, achievable specific activity, and retained affinity of the $\mathrm{mAb}$ postconjugation. This requires quantification of the number of covalently conjugated DFO moieties. The current methods of choice for quantification is accomplished by mass spectrometry methods or by isotope dilution assay. ${ }^{11-13}$ These methods can be time-consuming (a particular problem in clinical pharmacies), be costly, and/or lack accuracy, all of which may impede clinical translation of new immuno-PET agents significantly.

Herein we present a technology that enables real-time monitoring of coupling efficiency and rapid quantification of $\mathrm{mAb}$ functionalization with $\mathrm{DFO}$, while simultaneously rendering the conjugate suitable for bimodal imaging applications (PET and fluorescence imaging). This is accomplished by designing a bioorthogonal fluorogenic DFO probe that displays fluorescence turn-on upon ligation with a mAb-trans-cyclooctene (TCO). While examples of chelate-fluorophore conjugates have been reported, ${ }^{14-17}$ they do not provide direct monitoring of the conjugation reaction. Moreover, while PET provides essentially unlimited depth penetration, but limited resolution ( $\mathrm{mm}$ scale), optical techniques have resolution down to the sub- $\mu \mathrm{m}$ scale. Multimodal imaging agents can thus provide complementary information (beyond monitoring synthetic reaction conditions), particularly for subsequent intraoperative resection, margin determination of tumors, or endoscopic tattooing and biopsy. 


\section{RESULTS AND DISCUSSION}

Several synthetic approaches are possible for creating a multimodal tetrazine-dye conjugate. Among fluorescent probes, BODIPY derivatives represent a promising family due to their exceptional properties. They exhibit high stability, high extinction coefficients, sharp emission bands, and high quantum yields. ${ }^{18}$ With this in mind we chose our previously reported BODIPY-tetrazine conjugates as a starting point for multimodal probe development. These fluorogenic compounds display excellent fluorescence enhancement by utilizing through-bond energy transfer (TBET) as the main quenching mechanism in addition to good in vitro stability. Recently published BODIPY modification strategies that rely on modifications to the $\mathrm{BF}_{2}$ core proved to be a concise route to a stable conjugate that does not alter the fluorescence properties of the BODIPY core, yet retains high in vitro and in vivo inertness of the linker moiety. ${ }^{19,20}$ The original work done on alkoxy-substituted BODIPYs discovered that increasingly larger substituents were less prone to hydrolysis and displayed improved stability. ${ }^{20}$ We selected the commercially available 3-

hydroxycyclobutanecarboxylic acid as a suitable linker for its increased size compared to the original glycol-BODIPY derivatives ${ }^{20}$ Furthermore, the acid provides a synthetic handle for DFO conjugation. We began with the bioorthogonal fluorogenic BODIPY-Tz probe $\mathbf{1}$ in our synthesis (Scheme 1). ${ }^{21}$ To this end, the borenium intermediate generated by combining 1 and TMSOTf at $0{ }^{\circ} \mathrm{C}$ was quenched with 3-hydroxycyclobutanecarboxylic acid to give $\mathbf{2}$. Intermediate $\mathbf{2}$ was converted to the NHS activated ester 3 using $N, N$-disuccinimidyl carbonate and pyridine. Finally, NHS 3 was amidated with deferoxamine mesylate and $\mathrm{Et}_{3} \mathrm{~N}$ in DMF to give 4.

With bifunctional dye $\mathbf{4}$ in hand, we developed an efficient protocol to construct trastuzumab-TCO analogs with varying degrees of conjugation (see Experimental Procedures). Utilizing the fluorescence turn-on properties of $\mathbf{4}$, we successfully tracked the ligation between $\mathrm{mAb}-\mathrm{TCO}$ and $\mathbf{4}$ (Figure 1) to its rapid completion (under $3 \mathrm{~min}$ ). Having constructed several DFO-BODIPY-trastuzumab conjugates, we used UV/vis to determine the labeling efficiency and verified our results by a conventional characterization method, MALDI (Table 1).

We determined the absorbance spectrum of $\mathbf{4}$ after reaction with free trans-cyclooctenol to establish the A280/A500 ratio of the dye after tetrazine ligation. This correction factor allows quantification of the BODIPY contribution to the A280 of the conjugate, enabling deconvolution of the protein and dye concentrations from their characteristic extinction coeficients and calculation of the degree of labeling, per standard methods. Conjugate A was used for in vivo study based on its low labeling density and hence higher likeness to the native $\mathrm{mAb}$.

With the coupling method and quantification validated, we next determined the radiolabeling and in vitro cell binding properties of DFO-BODIPY-trastuzumab conjugate A. For this, we used a typical radiolabeling protocol starting from ${ }^{89} \mathrm{Zr}$-oxalate $(0.4 \mathrm{mCi})$ in $1 \mathrm{M}$ oxalic acid. After adjusting the $\mathrm{pH}$ of ${ }^{89} \mathrm{Zr}$-oxalate to 7.4-7.6, an aliquot of DFO-BODIPY-trastuzumab conjugate $\mathrm{A}(100 \mu \mathrm{g})$ was added and the reaction was gently mixed at room temperature for $1 \mathrm{~h}$, affording quantitative radiolabeling yield. The reaction was quenched and the ${ }^{89} \mathrm{Zr}$ 
labeled $\mathrm{mAb}$ was purified by size exclusion chromatography. The purified product ${ }^{89} \mathrm{Zr}$ DFO-BODIPY-trastuzumab was further characterized by radioTLC and gel electrophoresis (Figure 2). Comparison of Coomassie blue stained gel with autoradiography confirms association of the radiolabel with the antibody fragments.

In order to compare the new conjugation method to an established method of incorporating DFO onto antibodies, we synthesized a DFO-trastuzumab conjugate using conventional TFP-activation chemistry. The product was isolated and radiolabeled under analogous conditions as ${ }^{89} \mathrm{Zr}$-DFO-BODI-PY-trastuzumab, forming ${ }^{89} \mathrm{Zr}$-DFO-trastuzumab. Radiolabeling properties and obtained yields do not differ from the conventional ${ }^{89} \mathrm{Zr}-\mathrm{DFO}-$ trastuzumab analogue, which was synthesized as a reference.

Next, we compared specific binding of the mAb conjugate ${ }^{89} \mathrm{Zr}$-DFO-BODIPY-trastuzumab to HER2+/HER2- cells and compared its behavior to the conventional conjugate ${ }^{89} \mathrm{Zr}$-DFOtrastuzumab. We found that the two compounds exhibited very similar binding to HER2+ (BT474) and HER2- (BT20) cells in vitro (Figure 3), with significant differences between the binding obtained to HER2+ and HER2- cell lines for both compounds. The difference in binding of ${ }^{89} \mathrm{Zr}$-DFO-BODIPY-trastuzumab $(6.45 \pm 1.87 \%$ in BT474 versus $1.47 \pm 0.39 \%$ in BT20) versus ${ }^{89} \mathrm{Zr}$-DFO-trastuzumab $(5.48 \pm 0.45 \%$ in BT474 versus $0.75 \pm 0.21 \%$ in BT20) in HER2+ cells was found to be nonsignificant. Average nonspecific binding to BT20 is elevated for ${ }^{89} \mathrm{Zr}$-DFO-BODIPY-trastuzumab, possibly caused by the enhanced lipophilicity of the BODIPY-tetrazine fragment.

With the in vitro experiments providing satisfactory information on the performance of ${ }^{89} \mathrm{Zr}$ DFO-BODIPY-trastuzumab, we aimed to evaluate the bimodal probe in vivo. We generated tumor xenografts in female nude mice using HER2+ and HER2- cell lines for in vivo probe validation. Mice were injected with either probe and imaged 24, 48, 72, and $96 \mathrm{~h}$ after probe administration, followed by full biodistribution analysis after the last imaging time point.

We observed consistently enhanced target specific uptake of ${ }^{89} \mathrm{Zr}$-DFO-BODIPYtrastuzumab, yielding high tumor conspicuity that is evident on examination of PET images (Figure 4). Biodistribution data showed that both compounds behaved similarly in mice with respect to uptake in nontarget tissues, and no significant difference was obtained with respect to uptake in HER2 negative-tumors ( $p>0.5,14.14 \pm 8.23 \% \mathrm{ID} / \mathrm{g}$ for ${ }^{89} \mathrm{Zr}$-DFOtrastuzumab, $16.39 \pm 9.79 \% \mathrm{ID} / \mathrm{g}$ for ${ }^{89} \mathrm{Zr}$-DFO-BODIPY-trastuzumab). In mice bearing HER2+ tumors, we found very similar off-target behavior with both conjugates (Table 2). For tumor uptake, however, there was a remarkable 4-fold higher uptake in these cohorts ( $p$ $<0.0001,{ }^{89} \mathrm{Zr}$-DFO-BODIPY-trastuzumab: $237.27 \pm 28.90 \% \mathrm{ID} / \mathrm{g},{ }^{89} \mathrm{Zr}$-DFO-trastuzumab: $59.39 \pm 17.76 \% \mathrm{ID} / \mathrm{g})$.

Studies toward a better understanding of the cause of this exceptionally high probe uptake are ongoing. We hypothesize that the enhanced lipophilicity introduced by BODIPY contributes to increased retained activity over time. More specifically, it has been observed that BODIPY in addition to other dyes can stain lipophilic compartments such as the endoplasmic reticulum and golgi membranes. ${ }^{22}$ The effect of greater lipophilicity of ${ }^{89} \mathrm{Zr}$ DFO-BODIPY-trastuzumab is also apparent from the enhanced liver uptake when compared 
with liver uptake obtained for ${ }^{89} \mathrm{Zr}$-DFO-trastuzumab. The modified coupling method utilized for the incorporation of DFO does not have an effect on ${ }^{89} \mathrm{Zr}$ release, as the average uptake in bone is not statistically different in any of the cohorts investigated.

To evaluate the potential of the probe as a way to identify targets intraoperatively through fluorescence, we also acquired ex vivo fluorescence images of selected organs and tissues: liver, bone, muscle, and tumor (HER2+ and HER2- tumor) and compared them with ex vivo PET images of the same samples. We found that even after $96 \mathrm{~h}$, the fluorescence of BODIPY is found almost exclusively in HER2+ tumor tissue, indicating that the fluorophore remains fully associated and functional even after extended times of circulation and presence within the tumor tissue (Figure 5). These observations are of considerable translational importance. There is renewed interest in using optical techniques for tumor resection, margin delineation, targeting biopsies, and tumor monitoring by optical means.

\section{CONCLUSIONS}

In summary, we have developed a novel bioorthogonal fluorogenic labeling strategy for creating multimodal PET/ fluorescence mAb imaging probes. This represents the first example where a fluorogenic probe is used for reaction monitoring and quantification of a bioconjugation product. The fluorescence turn-on occurs during the conjugation reaction of biomolecule and fluorophore-chelator and provides a simple means of real time reaction monitoring as well as precise quantification of the number of conjugated chelators (DFO) by end point absorbance measurements. The resulting conjugate does not differ significantly from the conventional chelator-antibody conjugates in terms of radiolabeling properties and in vitro cell binding. Once administered to mice bearing tumor xenografts, the probe displays high target specificity as visualized by PET imaging at different time points, and robust ex vivo fluorescent imaging capabilities. We believe this broadly applicable conjugation strategy will find application with the use of near-infrared fluorophores for whole body NIR imaging in addition to other mAb's for increased target diversity.

\section{EXPERIMENTAL PROCEDURES}

\section{Chemicals and Reagents}

All reagents were purchased from Sigma-Aldrich and used without further purification unless otherwise noted.

\section{Instrumentation}

${ }^{1} \mathrm{H}$ and ${ }^{13} \mathrm{C}$ nuclear magnetic resonance spectra were recorded on a Bruker Ascend $400 \mathrm{MHz}$ spectrometer. MALDI data was acquired on a Bruker microflex MALDI-TOF MS. Silica Gel $60(40-63 \mu \mathrm{m})$ was used for flash column purification. High performance liquid chromatography-mass spectrometry analysis (HPLC-MS) was performed with a Waters instrument equipped with a Waters 2424 ELS Detector, Waters 2998 UV-vis Diode array Detector, Waters 2475 Multiwavelength Fluorescence Detector, and a Waters 3100 Mass Detector. Separations employed Waters XTerra RP C18 $5 \mu \mathrm{m}$ or Waters XSelect CSH Fluoro-Phenyl $2.5 \mu \mathrm{m}$ column, with a water:acetonitrile solvent gradient ( $0.1 \%$ formic acid). Fluorescence measurements were conducted with a QuantaMaster 400 fluorimeter (PTI, 
New Jersey, USA), and UV-vis absorption spectra on a HORIBA Dual-FL spectrophotometer.

\section{Synthetic Procedures. Preparation of 1}

To $p$-cyanophenyl-BODIPY ( $250.0 \mathrm{mg}, 0.71 \mathrm{mmol})$ in a microwave reaction tube under a stream of argon was added $\mathrm{Zn}(\mathrm{OTf})_{2}(130.1 \mathrm{mg}, 0.35 \mathrm{mmol}), \mathrm{MeCN}(0.38 \mathrm{~mL}, 7.16 \mathrm{mmol})$, 1,4-dioxane $(0.55 \mathrm{~mL})$, and $\mathrm{NH}_{2} \mathrm{NH}_{2}(1.12 \mathrm{~mL}, 35.8 \mathrm{mmol})$. The vessel was sealed and allowed to stir at $40{ }^{\circ} \mathrm{C}$ for $20 \mathrm{~h}$ after which it was allowed to cool and the septum removed. To the reaction mixture was added $\mathrm{NaNO}_{2}(988.1 \mathrm{mg}, 14.32 \mathrm{mmol})$ in $20 \mathrm{~mL}$ of water followed by $1 \mathrm{M} \mathrm{HCl}$ until the $\mathrm{pH}=3$. The aqueous phase was extracted three times with $\mathrm{CH}_{2} \mathrm{Cl}_{2}(100 \mathrm{~mL})$. The combined organic extracts were dried with $\mathrm{MgSO}_{4}$ and concentrated using a rotary evaporator. The crude mixture was purified using flash column chromatography (methylene chloride) to give $\mathbf{1}(63.2 \mathrm{mg}, 0.15 \mathrm{mmol}, 21.2 \%)$ as a dark red solid. $^{21}$

\section{Preparation of 2}

To 1 (273.1 mg, $0.65 \mathrm{mmol}$ ) in $5 \mathrm{~mL}$ of methylene chloride at $0{ }^{\circ} \mathrm{C}$ was added TMSOTf $(0.35 \mathrm{~mL}, 1.96 \mathrm{mmol})$ in $1 \mathrm{~mL}$ of methylene chloride over $30 \mathrm{~s}$. After $2 \mathrm{~min}, 3-$ hydroxycyclo-butanecarboxylic acid $(758.2 \mathrm{mg}, 6.53 \mathrm{mmol})$ and $N, N$-diisopropylethylamine $(0.57 \mathrm{~mL}, 3.26 \mathrm{mmol})$ dissolved in $3 \mathrm{~mL}$ of methylene chloride were added over a period of $2 \mathrm{~min}$ and the reaction taken out of $0{ }^{\circ} \mathrm{C}$ bath and allowed to stir at room temperature for 15 $\mathrm{min}$. The crude mixture was concentrated using a rotary evaporator and purified using flash column chromatography (methylene chloride:methanol, 10:0.2, $R_{\mathrm{f}}=0.23$ ) to give $\mathbf{2}$ (134.7 $\mathrm{mg}, 0.26 \mathrm{mmol}, 40.3 \%) .{ }^{1} \mathrm{H} \mathrm{NMR}\left(400 \mathrm{MHz}, \mathrm{CDCl}_{3}\right) \delta 8.74(\mathrm{~m}, 2 \mathrm{H}), 7.53(\mathrm{~d}, J=8.8 \mathrm{~Hz}$, 2H), 5.99 (s, 2H), 3.55 (p, $J=8.0 \mathrm{~Hz}, 1 \mathrm{H}), 3.12(\mathrm{~s}, 3 \mathrm{H}), 2.55(\mathrm{~s}, 6 \mathrm{H}), 2.47(\mathrm{~m}, 1 \mathrm{H}), 2.16(\mathrm{~m}$, 2H), 2.07 (m, 2H), 1.43 (s, 6H); ${ }^{13} \mathrm{C}$ NMR (100 MHz, MeOD) $\delta 179.0,169.3,165.1,157.7$, 144.4, 142.3, 140.7, 134.7, 132.9, 130.8, 130.7, 129.9, 129.8, 122.9, 63.1, 36.9, 33.2, 21.3, 15.2, 15.0. ESIMS [M-H] ${ }^{-}$calcd for $\mathrm{C}_{27} \mathrm{H}_{27} \mathrm{BFN}_{6} \mathrm{O}_{3}$ 513.22, found 513.20.

\section{Preparation of 3}

To $2(85.0 \mathrm{mg}, 0.165 \mathrm{mmol})$ in $5 \mathrm{~mL}$ of methylene chloride was added $N$, $N$-disuccinimidyl carbonate $(338.6 \mathrm{mg}, 1.32 \mathrm{mmol})$ and pyridine $(0.053 \mathrm{~mL}, 0.66 \mathrm{mmol})$. The reaction was allowed to stir at room temperature overnight after which it was concentrated using a rotary evaporator and purified using flash column chromatography (methylene chloride:methanol, 10:0.25, $\left.R_{\mathrm{f}}=0.33\right)$ to give $3(73.4 \mathrm{mg}, 72.8 \%) .{ }^{1} \mathrm{H} \mathrm{NMR}\left(400 \mathrm{MHz}, \mathrm{CDCl}_{3}\right) \delta 8.75(\mathrm{~m}, 2 \mathrm{H})$, $7.55(\mathrm{~m}, 2 \mathrm{H}), 5.99(\mathrm{~s}, 2 \mathrm{H}), 3.61(\mathrm{p}, J=8.0 \mathrm{~Hz}, 1 \mathrm{H}), 3.12(\mathrm{~s}, 3 \mathrm{H}), 2.79(\mathrm{bs}, 4 \mathrm{H}), 2.72(\mathrm{~m}$, $1 \mathrm{H}), 2.55(\mathrm{~s}, 6 \mathrm{H}), 2.26(\mathrm{~m}, 4 \mathrm{H}), 1.44(\mathrm{~s}, 6 \mathrm{H}) ;{ }^{13} \mathrm{C} \mathrm{NMR}\left(100 \mathrm{MHz}, \mathrm{CD}_{2} \mathrm{Cl}_{2}\right) \delta 170.6,169.8$, 169.3, 168.3, 164.3, 156.8, 143.2, 140.9, 140.0, 133.4, 131.9, 130.0, 129.9, 129.1, 129.0, 122.1, 62.2, 36.9, 27.6, 26.2, 26.1, 21.6, 15.2, 15.0. ESIMS $[\mathrm{M}+\mathrm{Na}]^{+}$calcd for $\mathrm{C}_{31} \mathrm{H}_{31} \mathrm{BFN}_{7} \mathrm{NaO}_{5}$ 634.22, found 634.24.

\section{Preparation of 4}

A solution of deferoxamine mesylate $(0.099 \mathrm{mmol}, 64.9 \mathrm{mg})$ and triethylamine $(20.5 \mu \mathrm{L}$, $0.15 \mathrm{mmol}$ ) in $0.25 \mathrm{~mL}$ dimethyl sulfoxide was added to $3(15.1 \mathrm{mg}, 0.025 \mathrm{mmol})$ in $0.1 \mathrm{~mL}$ 
of dimethyl sulfoxide. After stirring for 30 min the reaction was loaded directly on a $5 \mathrm{~g} \mathrm{C}_{18}$ Waters Sep-Pak and purified (compound 4 eluted off with $\mathrm{H}_{2} \mathrm{O}: \mathrm{MeCN}$ 17:7) to give 4 (12.1 $\mathrm{mg}, 46.5 \%) .{ }^{1} \mathrm{H}$ NMR $\left(400 \mathrm{MHz}, \mathrm{CDCl}_{3}\right) \delta 8.73(\mathrm{~m}, 2 \mathrm{H}), 7.53(\mathrm{~m}, 2 \mathrm{H}), 5.98(\mathrm{~s}, 2 \mathrm{H}), 3.61$ (m, 5H), $3.51(\mathrm{~m}, 1 \mathrm{H}), 3.17(\mathrm{~m}, 4 \mathrm{H}), 3.12(\mathrm{~s}, 3 \mathrm{H}), 2.79(\mathrm{~m}, 2 \mathrm{H}), 2.59(\mathrm{~m}, 4 \mathrm{H}), 2.54(\mathrm{~s}, 6 \mathrm{H})$, 2.32 (m, 2H), 2.17 (m, 2H), 2.09 (m, 2H), 1.98 (m, 2H), 1.56 (bs, 20H), 1.43 (s, 6H), 1.42 (s, $3 \mathrm{H})$. ESIMS $[\mathrm{M}-\mathrm{H}]^{-}$calcd for $\mathrm{C}_{52} \mathrm{H}_{73} \mathrm{BFN}_{12} \mathrm{O}_{10} 1055.57$, found 1055.80 .

\section{Preparation of MALDI-TOF Samples}

MALDI samples were prepared by drying a $1 \mu \mathrm{L}$ solution of a 2:1 mixture of antibody (150 $\mu \mathrm{g} / \mathrm{mL}$ ) in saline:sinapinic acid solution (1 mg sinapinic acid in $0.1 \mathrm{~mL}$ of $7: 3 \mathrm{MeCN}: \mathrm{H}_{2} \mathrm{O}$ ) on a MALDI target plate.

\section{Antibody Preparation for Labeling}

Trastuzumab, lyophilized powder for intravenous injection (Genentech), was dissolved in phosphate buffered saline with $100 \mathrm{mM}$ sodium bicarbonate (PBS-Bicarb, $\mathrm{pH}$ 8.6) at 5 $\mathrm{mg} / \mathrm{mL}$ (inclusive of the additives for preservation). The antibody solution was then buffer exchanged with a $40 \mathrm{~K}$ Zeba Spin column (ThermoFisher,USA) that had been equilibrated in the same buffer, per the manufacturer's protocol to remove preservatives and establish optimal $\mathrm{pH}$ for labeling. The absorbance of the antibody solution at $280 \mathrm{~nm}$ (A280) was measured by spectrophotometer (NanoDrop, Thermo Scientific) and converted to a molar concentration based on an extinction coefficient of $225000 \mathrm{M}^{-1} \mathrm{~cm}^{-1}$ for trastuzumab. Dimethylformamide was then added to a concentration $10 \%$ (v/v), and the solution was aliquoted into individual labeling reactions.

\section{Antibody-TCO Labeling and Initial Characterization}

Transcyclooctene- $\mathrm{PEG}_{4}$-NHS (ClickChemistryTools, Scotts-dale, AZ) was dissolved in dry DMF at a concentration of $10 \mathrm{mg} / \mathrm{mL}$ and added to the antibody solution in aliquots to obtain the correct labeling ratio, in moles of TCO per mole of antibody, for 2.5 and 10 equiv of TCO. For optimal labeling, antibody concentration in the final reaction should be between 2.5 and $3 \mathrm{mg} / \mathrm{mL}$. The labeling mixture was incubated at $25^{\circ} \mathrm{C}$ in a vortexing mixer for 30 min and then the reaction terminated by desalting on $40 \mathrm{~K}$ ZebaSpin columns that had been pre-equilibrated with $\mathrm{PBS}$ at $\mathrm{pH}$ 7.4, per the manufacturer's protocol. The concentration of labeled antibody and adequacy of DMF removal in the desalting columns was verified by Nanodrop measurement of A280 and of A260/280 ratio. The A260/A280 ratio is $<0.49$ for trastuzumab in buffer. In the event that higher A260/A280 ratios were observed, reflecting traces of DMF, the material was passed over an additional desalting column and reassessed. Labeled antibody solutions in PBS were stored at $4{ }^{\circ} \mathrm{C}$.

\section{Preparation of DFO-BODIPY-Trastuzumab}

Trastuzu-mab-TCO solutions in PBS were prepared for labeling by addition of 10\% DMF $(\mathrm{v} / \mathrm{v})$ to ensure solubility of the Tz-BODIPY-DFO conjugate 4 . A $10 \mathrm{mM}$ stock solution of 4 in DMF was prepared and added to the trastuzumab-TCO to reach a total molar ratio of 20 equiv of $\mathbf{4}$ per trastuzumab. The reaction was allowed to proceed at room temperature for 30 min and then desalted by successive passage over two $40 \mathrm{~K}$ ZebaSpin columns, to ensure 
complete removal of any free dye molecules. The concentration of labeled DFO-BODIPYtrastuzumab and the degree of labeling were assessed by spectrophotometry per standard methodology, using an A280/ A500 correction factor of 0.15 (unpublished data) to account for the contribution of the dye to the observed A280. The use of 0.15 as the correction factor reflects the ratio at the time point at which antibody labeling was assessed.

\section{Real-Time Monitoring of Tz-BODIPY-DFO Click Labeling}

A stock solution 4 in DMSO was diluted into $480 \mu \mathrm{L}$ of PBS to yield a final concentration of $12 \mu \mathrm{M}$, and then added to a microvolume quartz cuvette in a QuantaMaster 400 fluorimeter (PTI, New Jersey, USA). The sample was excited at $490 \mathrm{~nm}$ and the emission monitored at $510 \mathrm{~nm}$ in a continuous time series. To this solution was added $20 \mu \mathrm{L}$ of a $13.7 \mu \mathrm{M}$ stock of trastuzumab-TCO in PBS, for a net 25-fold dilution into PBS, and a final 20-fold excess of 4 relative to the antibody, demonstrating the feasibility of reaction monitoring by this method at even a high degree of labeling. Addition of the antibody produced an immediate rise in the fluorescence signal, after which the reaction was monitored to completion (no further fluorescence increase).

\section{Radiolabeling and Purification of DFO-BODIPY-tras-tuzumab}

${ }^{89} \mathrm{Zr}$ (oxalate) was received from Washington University at an average specific activity of (15 $\mathrm{mCi} / \mathrm{mL}$ ). For a typical radiolabeling procedure, an aliquot of $100 \mu \mathrm{L}$ was removed from the stock solution and transferred into a separate vial. The $\mathrm{pH}$ of the solution was adjusted with $1 \mathrm{M} \mathrm{Na}_{2} \mathrm{CO}_{3}(105 \mu \mathrm{L})$ to 7.4-7.6. The aliquot was mixed with $0.1 \mu \mathrm{g}$ ligand DFOtrastuzumab or DFO-BODIPY-trastuzumab (conjugate $\mathrm{A}$, in $100 \mu \mathrm{L}$ solution) and reacted under slow mixing for $1 \mathrm{~h}$. The reaction was monitored using radioTLC (solid phase: SigmaAldrich, Silica gel on TLC Aluminum foils, $10 \times 70 \mathrm{~mm}$; mobile phase; $50 \mathrm{mM}$ EDTA, $\mathrm{pH}$ 7). Under these conditions, ${ }^{89} \mathrm{Zr}$ (oxalate) migrates with the solvent front, while chelated ${ }^{89} \mathrm{Zr}$ remains at the origin. Complex solutions for in vivo administration were purified by size exclusion chromatography. The labeled product was characterized via radioTLC and gel electrophoresis.

\section{Cell Binding of Trastuzumab Conjugates}

Cell uptake experiments were performed in HER2+ (BT474), and HER2-(BT20) cells, in which $250 \mu \mathrm{L}$ of $10^{6}$ cells $/ \mathrm{mL}$ were seeded in 24-well plates. Cells were allowed to adhere overnight at $37{ }^{\circ} \mathrm{C}$ in a $5 \% \mathrm{CO}_{2}$ atmosphere. The media was removed and replaced with 125 $\mu \mathrm{L}$ of fresh complete media and $125 \mu \mathrm{L}$ of $50 \mathrm{ng} / \mathrm{mL}$ of ${ }^{89} \mathrm{Zr}$-DFO-BODIPY-trastuzumab or ${ }^{89} \mathrm{Zr}$-DFO-trastuzumab, saving aliquots in test tubes as a standard. The plates were incubated at $4{ }^{\circ} \mathrm{C}$ for $2 \mathrm{~h}$, gently rocking. The cells were washed three times with PBS, trypsinized, and transferred to test tubes. Radioactivity associated with cells was counted in a gamma counter. The percentage of bound radioactivity was calculated as the ratio of bound to the total radioactivity added per well multiplied by 100 .

\section{In Vivo, Ex Vivo, and Biodistribution (Xenograft Model)}

All animal experiments were conducted according to the guidelines of the Institutional Animal Care and Use Committee (IACUC). In vivo PET imaging and biodistribution studies 
were conducted in nude mice (Charles River Laboratories, MA). Mice were inoculated with $100 \mu \mathrm{L}$ of $10^{6}$ cells (BT-474 or BT-20) suspended in 1:1 saline and matrigel with no artificial stimuli. Tumors were allowed to grow for 3-4 weeks until tumors were palpable. Tumor sizes ranged from $(52 \pm 10) \mathrm{mm}^{3}$ (BT474 cells) and $(290 \pm 50) \mathrm{mm}^{3}$ (BT20 cells). Mice were injected via tail-vein with $100 \mu \mathrm{L}$ of $10-20 \mu \mathrm{Ci}$ of ${ }^{89} \mathrm{Zr}$-DFO-BODIPY-trastuzumab or ${ }^{89} \mathrm{Zr}$-DFO-trastuzumab (specific activity $1.5-2.0 \mathrm{mCi} / \mathrm{mg} \mathrm{mAb}$ ) in saline. Micro-PET/CT imaging experiments were conducted on a Sofie Biosciences G4 PET scanner (Culver City, CA, USA), 10 min static microPET images were acquired under general anesthesia (isoflurane $/ \mathrm{O}_{2}$ ) at $24,48,72$, and $96 \mathrm{~h}$ post injection. Mice were sacrificed and organs were harvested, weighed, and assayed in the gamma counter for biodistribution studies. Radioactivity associated with each organ was expressed as percentage of injected dose per gram of organ (\% ID/g). Biodistribution data were assessed by unpaired tests using GraphPad Prism (version 6.02 for Windows GraphPad Software, San Diego, CA, USA) in order to determine any significant differences $(p<0.05)$. For ex vivo fluorescence imaging, an IVIS Spectrum imaging system was used to record the images (exposure time, $120 \mathrm{~s}$; bin $=8, f=1, \mathrm{FOV}=\mathrm{D})$. Selected organs and tissue samples were placed in culture dishes $(60$ $\mathrm{mm}$ diameter), and samples were excited at $465 \mathrm{~nm}$, with fluorescence emission monitored at $520 \mathrm{~nm}$.

\section{Supplementary Material}

Refer to Web version on PubMed Central for supplementary material.

\section{Acknowledgments}

We are grateful to Dr. Ralph Mazitschek for many helpful discussions, Nicholas Rotile for assistance with PET imaging, Shadi Esfahani and Umar Mahmood for assistance with the HER2+ cell line and Brijesh Bhayana for MALDI-TOF acquisition. The authors acknowledge funding by NIH RO1EB010011, RO1EB009062, 2P50A086355, and K99HL125728.

\section{References}

1. Rosenberg SA, Yang JC, Restifo NP. Cancer immunotherapy: moving beyond current vaccines. Nat Med. 2004; 10:909-915. [PubMed: 15340416]

2. Leavy O. Immunotherapy: A triple blow for cancer. Nat Rev Cancer. 2015; 15:258-259. [PubMed: 25833214]

3. Wu AM. Antibodies and antimatter: the resurgence of immuno-PET. J Nucl Med. 2008; 50:2-5. [PubMed: 19091888]

4. Van Dongen GA, Visser GW, Lub-de Hooge MN, De Vries EG, Perk LR. Immuno-PET: a navigator in monoclonal antibody development and applications. Oncologist. 2007; 12:1379-1389. [PubMed: 18165614]

5. Pandit-Taskar N, O’Donoghue JA, Durack JC, Lyashchenko SK, Cheal SM, Beylergil V, Lefkowitz RA, Carrasquillo JA, Martinez DF, Fung AM, Solomon SB, GM, Heller G, Loda M, Nanus DM, Tagawa ST, Feldman JL, Osborne JR, Lewis JS, Reuter VE, Weber WA, Bander NH, Scher HI, Larson SM, Morris MJ. A Phase I/II Study for Analytic Validation of 89Zr-J591 ImmunoPET as a Molecular Imaging Agent for Metastatic Prostate Cancer. Clin Cancer Res. 2015; 21:5277. [PubMed: 26175541]

6. Alfuraih A, Alzimami K, Ma AK, Alghamdi A, Al Jammaz I. Effective dose to immuno-PET patients due to metastable impurities in cyclotron produced zirconium-89. Radiat Phys Chem. 2014; 104:145-149. 
7. Zeglis BM, Lewis JS. The Bioconjugation and Radiosynthesis of 89Zr-DFO-labeled Antibodies. J Visualized Exp. 2015:e52521-e52521.

8. Ikotun OF, Marquez BV, Huang C, Masuko K, Daiji M, Masuko T, McConathy J, Lapi SE. Imaging the L-type amino acid transporter-1 (LAT1) with Zr-89 immunoPET. PLoS One. 2013; 8:e77476. [PubMed: 24143237]

9. Deri MA, Zeglis BM, Francesconi LC, Lewis JS. PET imaging with 89 Zr: from radiochemistry to the clinic. Nucl Med Biol. 2013; 40:3-14. [PubMed: 22998840]

10. Zeglis BM, Lewis JS. A practical guide to the construction of radiometallated bioconjugates for positron emission tomography. Dalton Trans. 2011; 40:6168-6195. [PubMed: 21442098]

11. Marquez BV, Ikotun OF, Zheleznyak A, Wright B, Hari-Raj A, Pierce RA, Lapi SE. Evaluation of 89Zr-pertuzumab in Breast Cancer Xenografts. Mol Pharmaceutics. 2014; 11:3988-3995.

12. Holland JP, Divilov V, Bander NH, Smith-Jones PM, Larson SM, Lewis JS. 89Zr-DFO-J591 for immunoPET of prostate-specific membrane antigen expression in vivo. J Nucl Med. 2010; 51:1293-1300. [PubMed: 20660376]

13. Holland JP, Caldas-Lopes E, Divilov V, Longo VA, Taldone T, Zatorska D, Chiosis G, Lewis JS. Measuring the pharmacodynamic effects of a novel Hsp90 inhibitor on HER2/neu expression in mice using 89 Zr-DFO-trastuzumab. PLoS One. 2010; 5:e8859. [PubMed: 20111600]

14. Pandey SK, Gryshuk AL, Sajjad M, Zheng X, Chen Y, Abouzeid MM, Morgan J, Charamisinau I, Nabi HA, Oseroff A. Multimodality agents for tumor imaging (PET, fluorescence) and photodynamic therapy. A possible "see and treat" approach. J Med Chem. 2005; 48:6286-6295. [PubMed: 16190755]

15. Lhenry D, Larrouy M, Bernhard C, Goncalves V, Raguin O, Provent P, Moreau M, Collin B, Oudot A, Vrigneaud JM, et al. BODIPY: A Highly Versatile Platform for the Design of Bimodal Imaging Probes. Chem - Eur J. 2015; 21:13091-13099. [PubMed: 26206262]

16. Brand C, Abdel-Atti D, Zhang Y, Carlin S, Clardy SM, Keliher EJ, Weber WA, Lewis JS, Reiner T. In vivo imaging of GLP-1R with a targeted bimodal PET/fluorescence imaging agent. Bioconjugate Chem. 2014; 25:1323-1330.

17. Zeglis BM, Davis CB, Abdel-Atti D, Carlin SD, Chen A, Aggeler R, Agnew BJ, Lewis JS. Chemoenzymatic Strategy for the Synthesis of Site-Specifically Labeled Immunoconjugates for Multimodal PET and Optical Imaging. Bioconjugate Chem. 2014; 25:2123-2128.

18. Bernhard C, Goze C, Rousselin Y, Denat F. First bodipy-DOTA derivatives as probes for bimodal imaging. Chem Commun. 2009; 46:8267-8269.

19. Hendricks JA, Keliher EJ, Wan D, Hilderbrand SA, Weissleder R, Mazitschek R. Synthesis of [18F] BODIPY: bifunctional reporter for hybrid optical/positron emission tomography imaging. Angew Chem, Int Ed. 2012; 51:4603-4606.

20. Courtis AM, Santos SA, Guan Y, Hendricks JA, Ghosh B, Szantai-Kis DM, Reis SA, Shah JV, Mazitschek R. Monoalkoxy BODIPYs - A Fluorophore Class for Bioimaging. Bioconjugate Chem. 2014; 25:1043-1051.

21. Carlson JC, Meimetis LG, Hilderbrand SA, Weissleder R. BODIPY-Tetrazine Derivatives as Superbright Bioorthogonal Turn-on Probes. Angew Chem, Int Ed. 2013; 52:6917-6920.

22. Cunningham CW, Mukhopadhyay A, Lushington GH, Blagg BS, Prisinzano TE, Krise JP. Uptake, distribution and diffusivity of reactive fluorophores in cells: implications toward target identification. Mol Pharmaceutics. 2010; 7:1301-1310. 


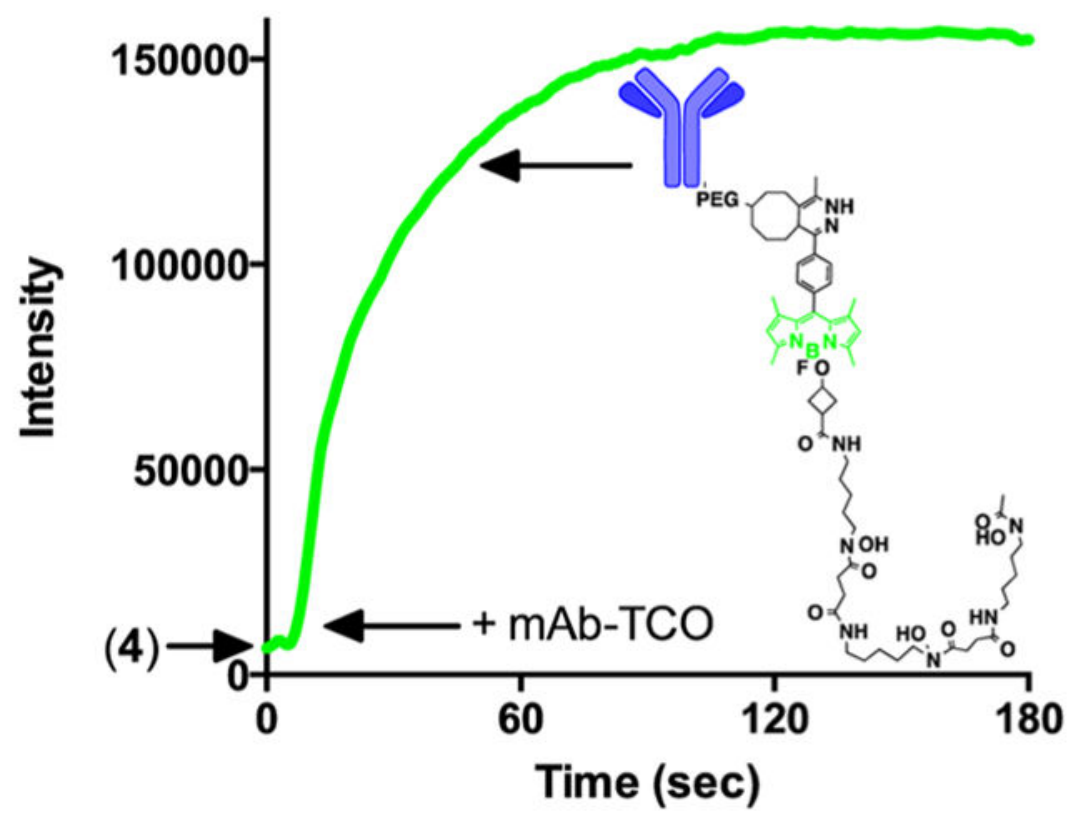

Figure 1.

Fluorescence turn on observation during the reaction between trastuzumab-TCO and TzBODIPY-DFO 4 to yield DFO-BODIPY-trastuzumab. 


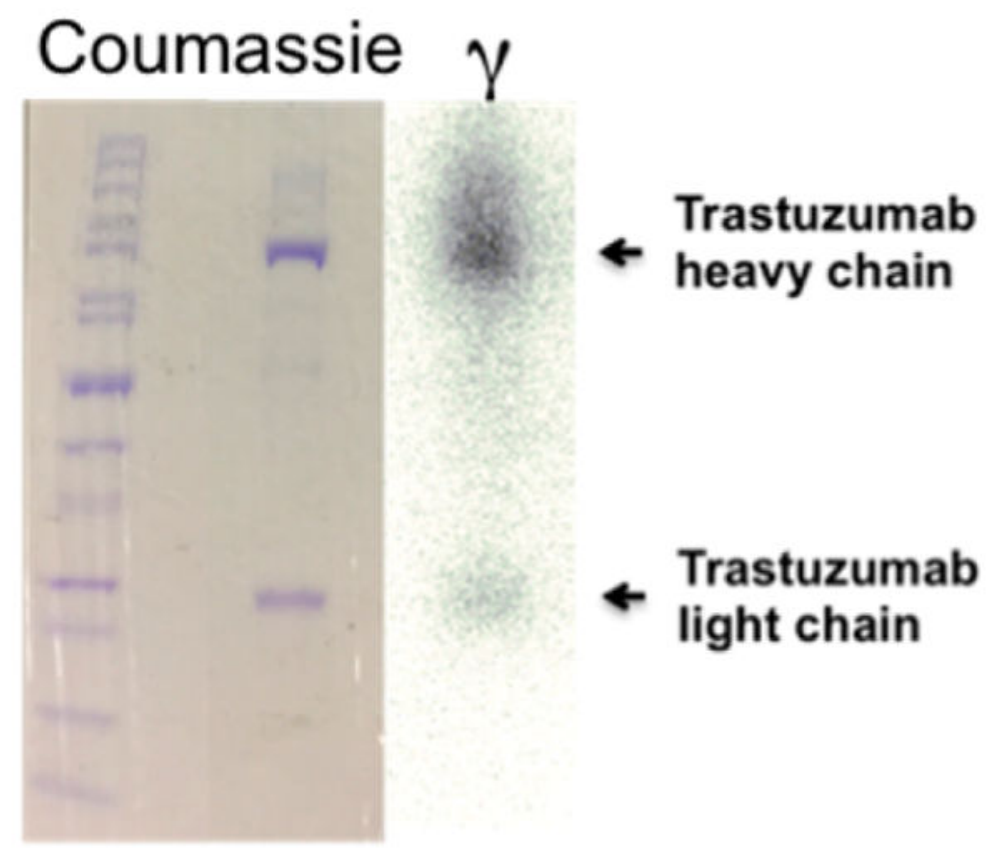

Figure 2.

Gel electrophoresis of ${ }^{89} \mathrm{Zr}$-DFO-BODIPY-trastuzumab, showing heavy and light chain both in the photograph obtained after coumassie protein staining, as well as image obtained from autoradiography of identical gel, indicating association of the radioactive label with the mAb fragments. 


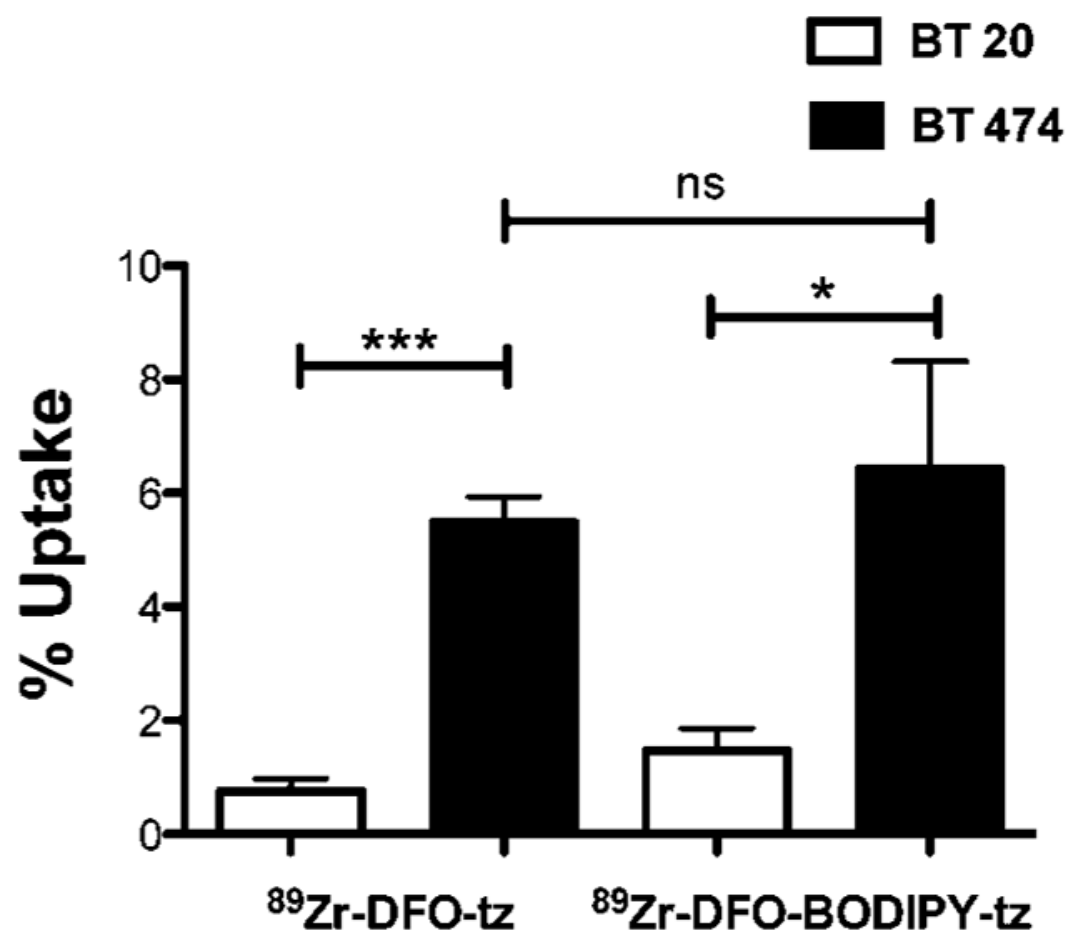

Figure 3.

Quantification of cell uptake of trastuzumab (tz) conjugates shows significant difierence in uptake in HER2+ cells versus HER2-cells for both ${ }^{89} \mathrm{Zr}$-DFO-BODIPY-trastuzumab and ${ }^{89} \mathrm{Zr}$-DFO-trastuzumab $(n=4)$. 

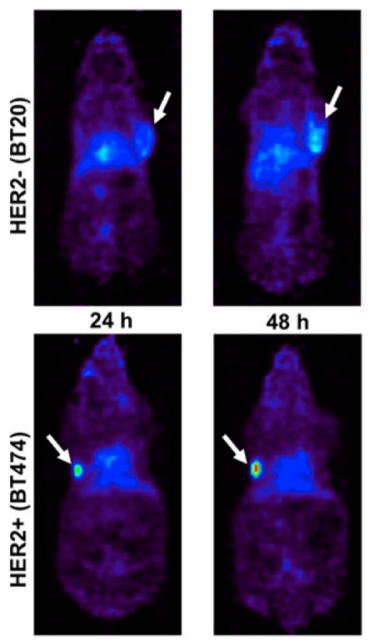

${ }^{89}$ Zr-DFO-BODIPY-trastuzumab

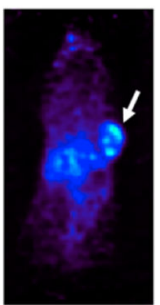

$72 \mathrm{~h}$

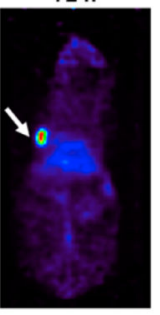

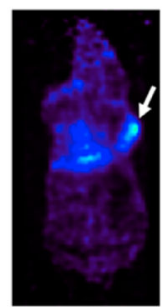

$96 \mathrm{~h}$
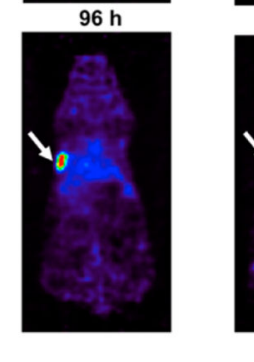

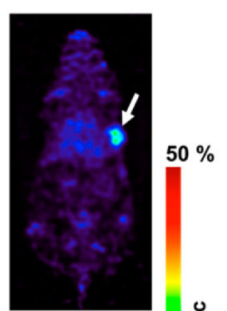

$96 \mathrm{~h}$

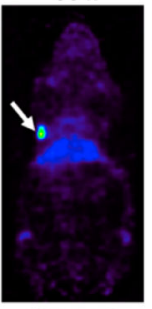

${ }^{89} \mathrm{Zr}$-DFO-

trastuzumab

Figure 4.

Coronal images of mice with BT20 and BT474 xenografts obtained with ${ }^{89} \mathrm{Zr}$-DFOBODIPY-trastuzumab. The images display low nonspecific uptake in the BT20 tumor (HER2-), at all imaging time points in contrast with high specific uptake in BT474 xenografts (HER2+), increasing over time. The arrow indicates the location of the tumor. 


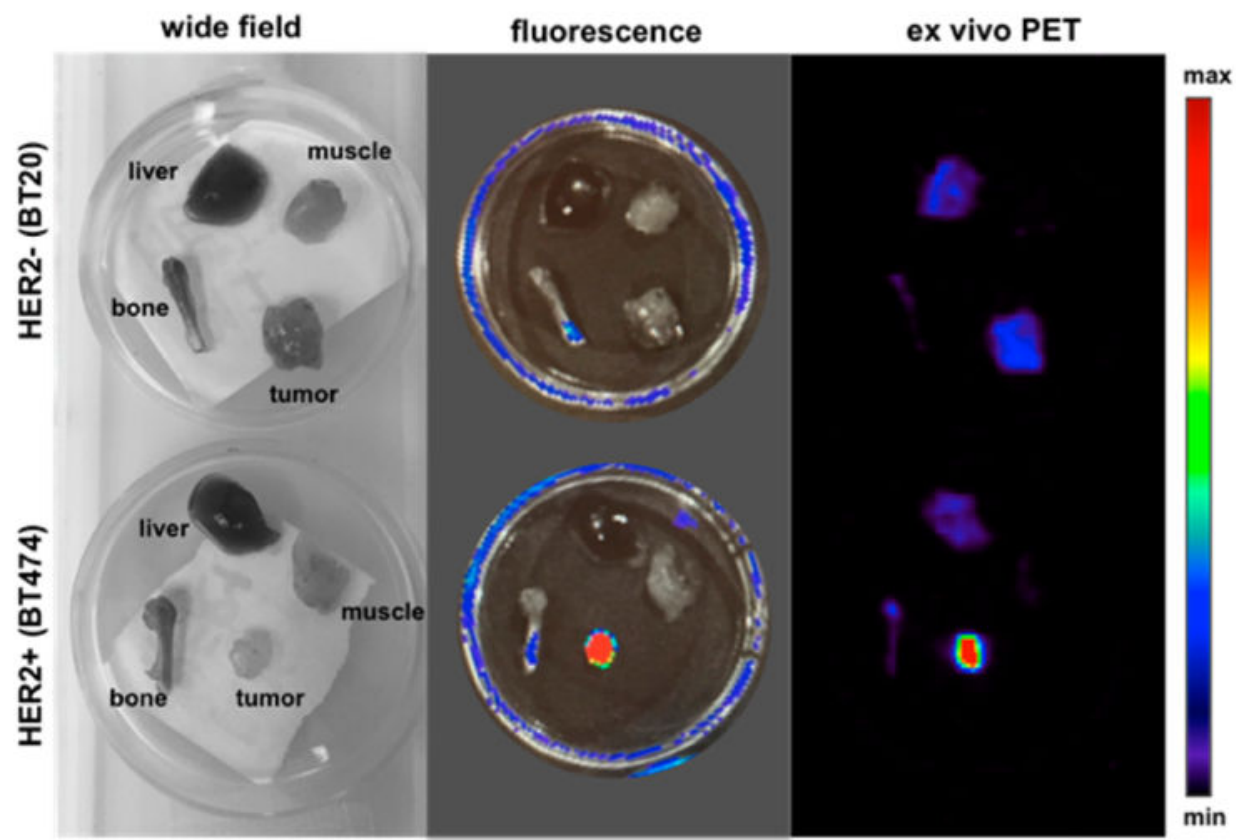

Figure 5.

Wide field, fluorescence (465ex/520em) and ex vivo PET images acquired of muscle, liver, bone, and tumor. The enhanced uptake of the fluorescent immunoconjugate in HER2+ tumor tissue when compared with HER2- tumor tissue is evident. 


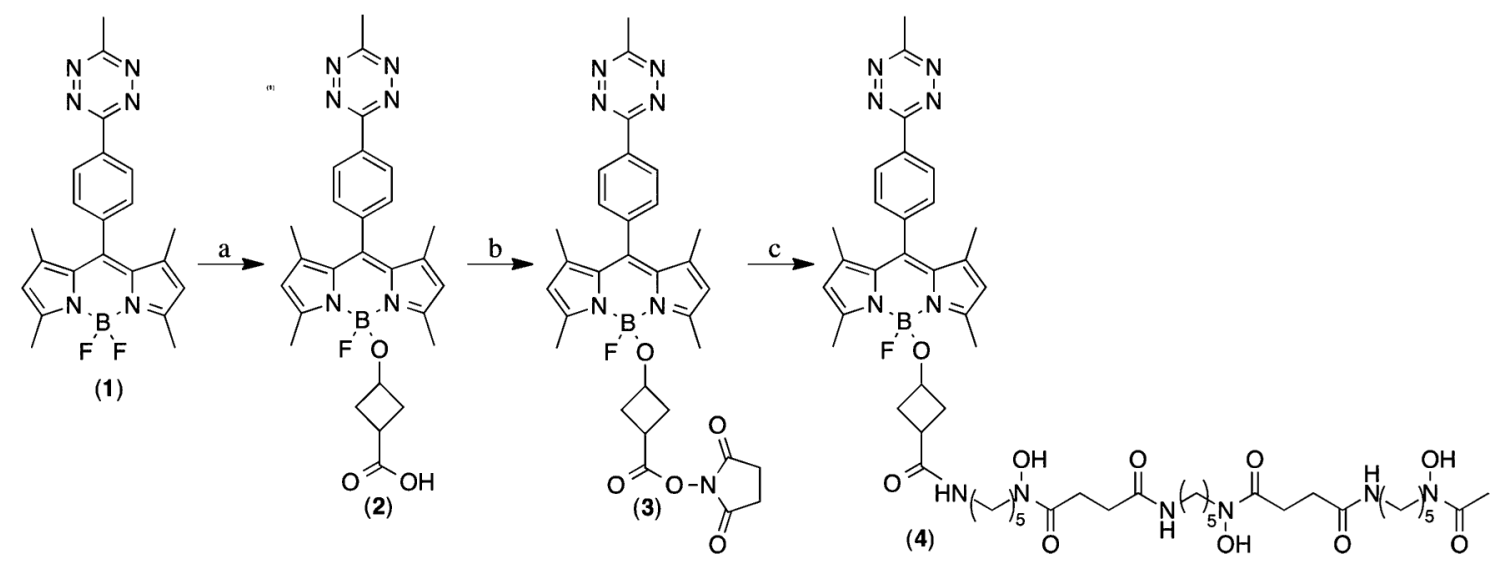

Scheme 1.

Synthesis of Bioorthogonal Fluorogenic PET Probe (4) ${ }^{a}$

${ }^{a}$ Reagents and conditions: (a) TMSOTf at $0{ }^{\circ} \mathrm{C}$ for 2 min in DCM, followed by 3 hydroxycyclobutanecarboxylic acid and DIPEA; (b) DSC and pyridine in DCM at rt; (c) Deferoxamine mesylate and $\mathrm{Et}_{3} \mathrm{~N}$ in $\mathrm{DMF}$ at $\mathrm{rt}$. 


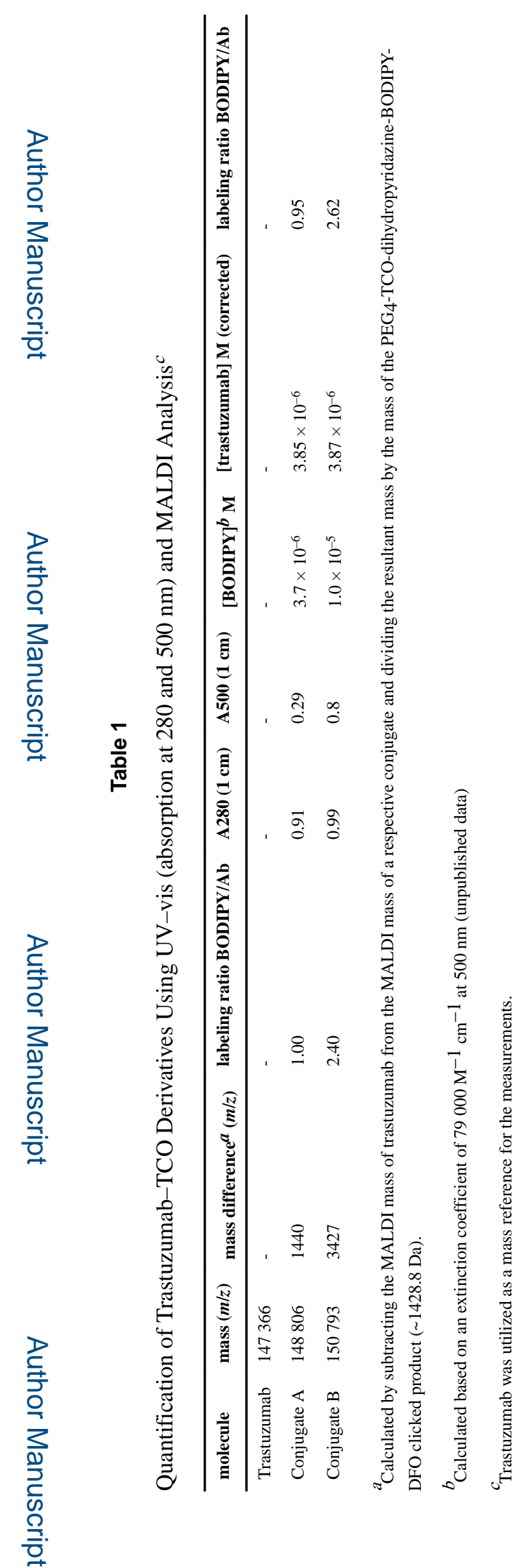

Bioconjug Chem. Author manuscript; available in PMC 2016 May 05. 


\section{Table 2}

Biodistribution of ${ }^{89}$ Zr-DFO-BODIPY-Trastuzumab $(n=4)$ and ${ }^{89}$ Zr-DFO-BODIPY-Trastuzumab $(n=5)$ at 96 h p.i. in Nude Mice Bearing BT20 or BT474 Xenografts ${ }^{a}$

\begin{tabular}{|c|c|c|c|c|}
\hline & \multicolumn{2}{|c|}{ mice with BT20 xenografts } & \multicolumn{2}{|c|}{ mice with BT474 xenografts } \\
\hline & ${ }^{89} \mathrm{Zr}$-DFO-trastuzumab & ${ }^{89} \mathrm{Zr}$-DFO-BODIPY-trastuzumab & ${ }^{89} \mathrm{Zr}$-DFO-trastuzumab & ${ }^{89} \mathrm{Zr}$-DFO-BODIPY-trastuzumab \\
\hline Blood & $6.96 \pm 2.91 \%$ & $8.03 \pm 4.49 \%$ & $8.73 \pm 1.22 \%$ & $10.08 \pm 6.70 \%$ \\
\hline Heart & $1.78 \pm 0.50 \%$ & $3.20 \pm 1.69 \%$ & $2.98 \pm 0.38 \%$ & $3.68 \pm 1.84 \%$ \\
\hline Lung & $4.01 \pm 3.41 \%$ & $4.87 \pm 2.67 \%$ & $3.13 \pm 1.84 \%$ & $9.13 \pm 7.41 \%$ \\
\hline Stomach & $0.76 \pm 0.36 \%$ & $0.73 \pm 0.46 \%$ & $0.71 \pm 0.12 \%$ & $1.65 \pm 0.75 \%$ \\
\hline Liver & $6.03 \pm 0.32 \%$ & $15.54 \pm 4.45 \%$ & $8.69 \pm 4.25 \%$ & $22.08 \pm 15.36 \%$ \\
\hline Intestine & $0.89 \pm 0.32 \%$ & $2.15 \pm 1.10 \%$ & $1.13 \pm 0.27 \%$ & $2.70 \pm 1.09 \%$ \\
\hline Kidney & $3.40 \pm 0.89 \%$ & $6.04 \pm 1.14 \%$ & $3.63 \pm 0.61 \%$ & $3.74 \pm 1.26 \%$ \\
\hline Bone & $3.83 \pm 1.91 \%$ & $3.82 \pm 1.63 \%$ & $2.85 \pm 2.18 \%$ & $3.51 \pm 2.40 \%$ \\
\hline Muscle & $0.27 \pm 0.09 \%$ & $0.46 \pm 0.25 \%$ & $0.30 \pm 0.18 \%$ & $0.32 \pm 0.24 \%$ \\
\hline Skin & $2.83 \pm 1.75 \%$ & $5.39 \pm 3.80 \%$ & $2.65 \pm 1.70 \%$ & $5.32 \pm 2.87 \%$ \\
\hline Tumor & $14.14 \pm 8.23 \%$ & $16.39 \pm 9.79 \%$ & $59.39 \pm 17.76 \%$ & $237.27 \pm 28.90 \%$ \\
\hline
\end{tabular}

